\title{
An Integrated Electronic Medical Record System (iEMRS) with Decision Support Capability in Medical Prescription
}

\author{
S.L. TING, W.H. IP, ALBERT H.C. TSANG, GEORGE T.S. HO \\ JOURNAL OF SYSTEMS AND INFORMATION TECHNOLOGY, 14(3), PP. 236 - 245
}

\begin{abstract}
Purpose: This study presents how a clinical decision support system can help in prescription and knowledge acquisition processes.

Design/methodology/approach: An integrated Electronic Medical Records System (iEMRS) is designed to enhance the decision support quality in prescription.

Findings: By evaluating the system performance through 135 prescription records collected from a Hong Kong medical organization, iEMRS shows a satisfactory result in suggesting medicines that is properly the same as the decisions made by the physicians.

Originality/value: Compared with the static clinical guidelines built (manually) in the traditional clinical decision support system, knowledge in iEMRS is generated by the knowledge discovery result from professional experiences of various physicians and patient histories, which are more dynamic in nature. A treatment algorithm, designed in data mining technique, is introduced to improve information management in medical organization by integration of decision support capability and EMRS, and supplement the deficiencies of traditional clinical decision support system.
\end{abstract}

Keywords: Data Mining; Decision Support; Electronic Medical Record; Medical Prescription.

\section{Introduction}

The Institute of Medicine defined Electronic Medical Records System (EMRS) as a longitudinal collection of electronic health information for and about persons in 2003. It allows electronic access to person- and population-level information by authorized users and supports efficient processes for health care delivery. A lot of medical organizations around the world have adopted EMRS, for example, in some Asian countries (like Singapore, Japan and Taiwan), EMRS has been adapted in a long time (Khanapi et al., 2008). Information and Communications Technology (ICT) approach to EMRS is accepted in hospitals as well as clinical functions and logistics activities to enhance the transmission of patient medical records and communication among physicians. 
In 1994, hospitals and clinics in Japan launched 'Health information Strategy 21' that processes EMRS with the mission to provide quality and efficient healthcare services. Although there is no government-centered EMRS, some of the local, regional and single hospitals have installed EMRS which allows data sharing among physicians (Toyoda, 1998). In Taiwan, an EMRS initiative was officially launched in 1995 under the National Information Infrastructure Project (Hu et al., 2002). Although the healthcare provisions in Taiwan are driven by the private sectors, the government still has authority for chiefly performing a regulatory role on health policy and strategy (Holliday and Tam, 2004). In Hong Kong, The Hong Kong Hospital Authority has supported the development and implementation of EMRS initiatives (Holliday and Tam, 2004; Cheung et al., 2001) in hospitals. All in all, these EMRS initiatives help medical organizations to save cost and raise medical services standard.

The prescription process starts from a diagnosis made by the physicians and ends with the distribution of drugs. In this process, physicians have to make use of their knowledge to give treatments. In case the prescription is not appropriate for patients, it harms the health of patients. Moreover, it harms the reputations and devastates the standard of medical services. In order to raise standard of medical services, medical organizations in many countries tend to apply EMRS that can enhance the integrity of information and cope with the problems of missing records. However, despite the widespread application of EMRS, the situation of medical prescription errors cannot be reduced (Condren et al., 2010). Attempted to address such inadequacy of EMRS, integration of the current system with prescription decision support capability is proposed in this study.

In this study, an integrated EMRS with prescription decision support capability, abbreviated as iEMRS, is proposed to combine the concept of clinical decision support system (CDSS) and traditional electronic medical record system in providing knowledge that can serve as references for quality decisions in prescription. The objective of iEMRS is to provide useful information to assist decision making during the prescription process. To the best of authors' knowledge, the current CDSS is mainly developed based on human efforts and experiences. However, with the increasing amount of information being captured in the EMRS, there is a pressing need to employ informatics technique to further analyze the clinical data for deriving the new clinical rules and guidelines. Furthermore, compared with the static clinical guidelines built (manually) in the traditional CDSS, knowledge in iEMRS is generated by knowledge discovery result from the professional experiences of various physicians and patient histories, which are more dynamic in nature. With numerous knowledge (i.e. prescription behaviors of different physicians) stored in the iEMRS, the quality of the prescription can significantly enhanced by considering the peer-based experiences (Ting et al., 2010; Ting et al., 2011). As a result, a treatment algorithm, designed in data mining technique, is introduced to improve the information management in medical organization by integration of decision support capability and EMRS, and supplement the deficiencies of traditional CDSS.

\section{Background Research}


The increasing demand for well-structured medical records contributes to the development of EMRS; in which it allows physicians to manage the patient information with computer tools effectively and efficiently. Comparing with paper-based medical records, EMRS benefits in improving the integrity and consistency of patient data, standardizing the medical report format, fastening communication among healthcare professions, securing treatment information, and reducing the overhead costs (Roukema et al., 2006). All these advantages facilitate the adopting of EMRS in hospitals and large-scare clinics over the world.

Although EMRS provides better management of information and quality of medical information, Condren et al. (2010) discuss that it is insufficient for reducing medical errors in prescription. As highlighted by Kozier et al. (2004), prescription is a process which requires a lot of consideration, such as demography factor, psychological factor, as well as illness and disease factor. In order to provide an accurate prescription, physicians have to consider the age, medical history, allergy, gender and diagnosis in each treatment (Regnstrom and Burgess, 2005). It is argued that the traditional EMRS (which is only used for data recording) lacks the capability in supplying medical knowledge that is critical for physicians’ diagnostic and therapeutic decision.

To support physicians in making decisions during the prescription process, integration of the concept of CDSS into the EMRS is proposed (Sánchez et al., 2005; Ting et al., 2010). In this integration, the EMRS can extend its function of electronic medical records by incorporating the clinical decision support capability. According to the study of Randolph et al. (1999), there are three main characteristics in such integrated approach: edit functions, record analysis functions and CDSS.

Edit functions are the fundamental functions in EMRS with decision support capability. Physicians can input and save the text of diagnosis, symptoms, treatment plan and personal information of patients into electronic format. When changes are encountered, physicians can change the information via EMRS interface that ensures necessary information is entered every time.

With the storage of clinical information in the system, records analysis functions allow healthcare professionals to analyze the data from databases for further uses. For example, according to Dangerous Drugs Ordinance, physicians have to record the patients' information (e.g. name and the date of visit) when the patients are being given the dangerous drugs. In this way, records analysis functions can sort the dangerous drugs information to physicians, so as to reduce the chance of dispensing incorrect mediation to the patients.

The final characteristic, CDSS, is a computer tool which uses two or more items of data to generate patient- or encounter-specific advice (Wyatt, 2000). It is typically designed to integrate a medical knowledge base, patient data and an inference engine to generate case specific advice and give opinions about the actions, such as altering, reminding, critiquing, and assisting, when a certain event occurs (Randolph et al., 1999). For example, alerting message is shown to notify physicians when they prescribe the wrong drugs to patient (like the case that the patient is allergic to the prescribed medicines). Another example is that CDSS can suggest physicians with alternative medicines which bring the same effect when they have to change the original decision (Teich et al., 2005). In case the 
stock of Drug X is not enough for the case, therefore CDSS will suggest Drug Y which has the same mediation effect (and suitable for the patients) to the physicians.

\section{System Architecture of iEMRS}

3.1 Presentation Tier: Presentation Tier is the media platform that consists of several devices used by nurses and physicians to access the system. To communicating the iEMRS, physicians and nurses must have their own computers for accessing the system. In addition to the computers, printers are employed for drugs' label printing and reporting printing as well. Presentation Tier is connected to a secure Communication Tier that the users can enquire the system and obtain the information like patient information, medical history of patients and drugs' information. When particular information a requested (by users), the Logic Tier is motivated to take the appropriate information from the Data Tier in iEMRS and display the information to the users.

3.2 Communication Tier: Communication Tier is supported by secure connection which can protect medical data privacy and integrity. It represents a link between the Presentation Tier and the Logic Tier. With the use of wired connection such as Local Area Network (LAN), computers inside the network can connect to the same data warehouse under the shared drive. As a result, real-time medical data transmission in operations can be achieved.

3.3 Logic Tier: Logic Tier is the layer that consists of the system objectives and decision rules for administrative management, message alert and knowledge generation. It is responsible for processing the requests from other tiers. In iEMRS, the Logic Tier is a medium among the Communication Tier, and the Data Tier. In Logic Tier, four major logics, namely Information Recording, Information Retrieval, Real Time Alert and Treatment Patterning Algorithm, would be determined to achieve the services goals. All these logics are introduced and discussed one by one in the below section. 


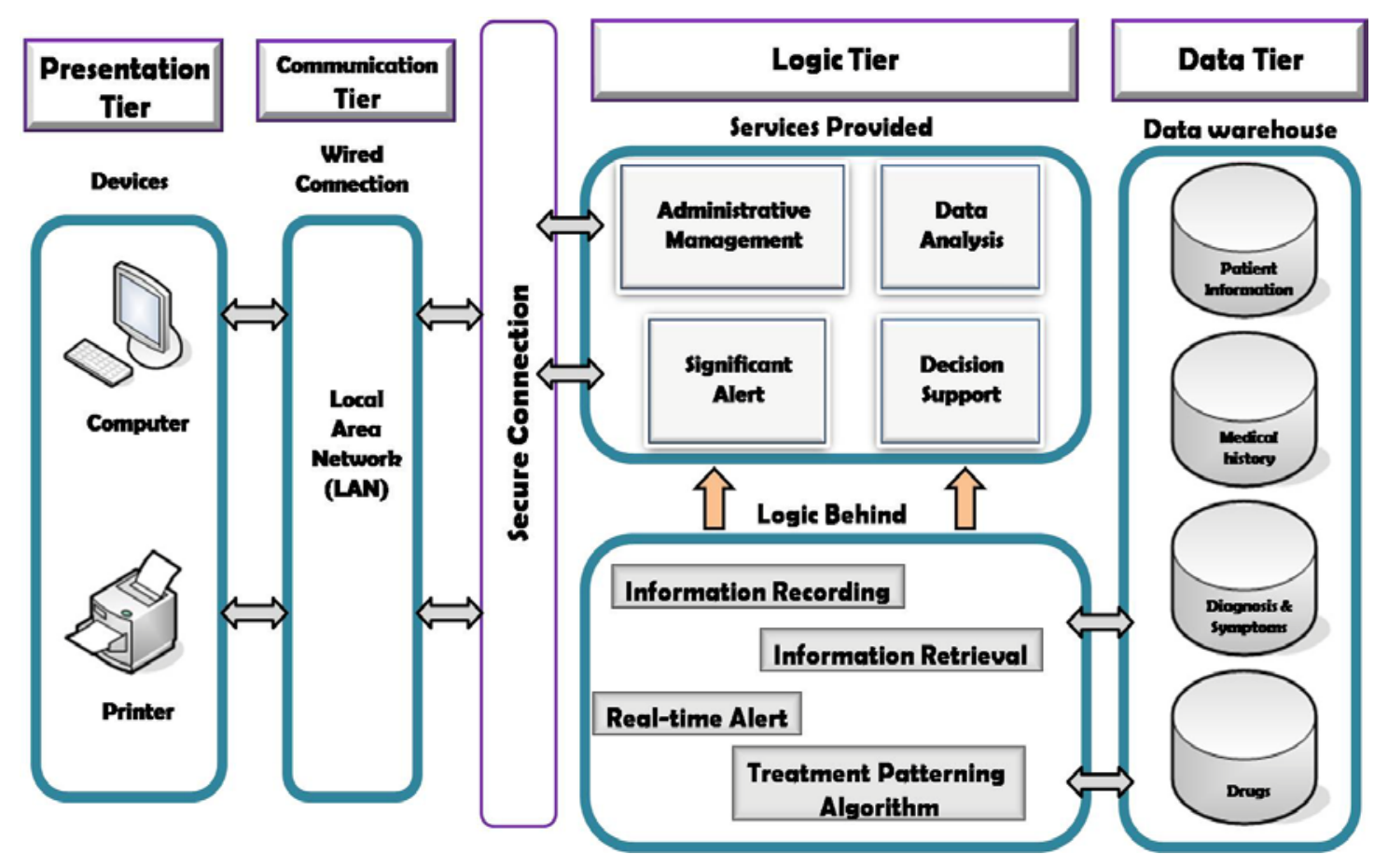

Figure 1. System architecture of iEMRS

3.3.1 Information Recording: The Logic Tier is responsible in recording the clinical information systematically. In each patient's visit, all his/her data are collected by the users (i.e. nurses and physicians) and hence recorded in the system and the data warehouse (of the Data Tier) in a standardized format for further analysis and decision rules generation.

3.3.2 Information Retrieval: Physicians can review the medical history of patients in legible formats via the Logic Tier. Such history can support physicians in making therapeutic decision with the medical knowledge discovered from the other physicians' diagnostic results and prescription behaviors. Furthermore, this logic can support to retrieve all the drugs available in the current medical organization (e.g. the drug name, cost, and description) so as to facilitate the medicine selection process. After the physicians decide the prescription plan, nurses can review it via the Logic Tier and print it out via Presentation Tier. Figure 2 depicts the overall mechanism of Information Retrieval.

3.3.3 Real Time Alert: In addition to above elements, Logic Tier also establishes Real Time Alert function. When the system receives any information which is contradicted to the original information in data warehouse of Data Tier, it will give an alarm message in Presentation Tier to notify the users that there is enquiry in the inputted information. This is particular useful in the case of medicine allergic as alert message will prompt out when the choice of prescribed drugs is not appropriate. For example, when the physician decides to prescribe Drug A for a patient, the property of Drug A will then be compared with the medical allergy history of the patient. If there is any allergic reaction discovered, an alert message is prompted to notify the physician that the drug will cause allergy effect. 


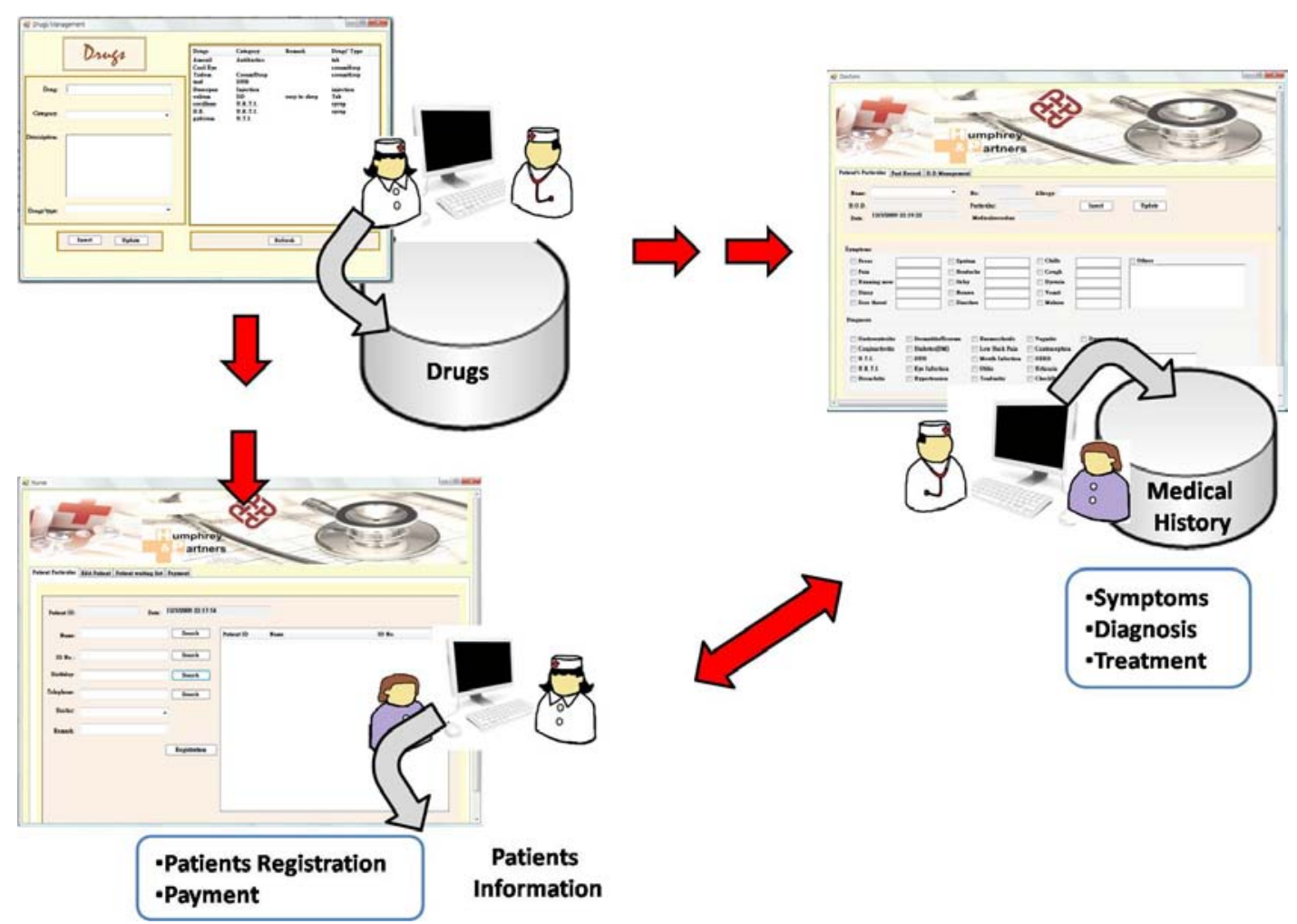

Figure 2. Information retrieval of Logic Tier in iEMRS

3.3.4 Treatment Patterning Algorithm: With better decision support in prescription process, the Logic Tier offers the Treatment Pattern Algorithm to provide knowledge for physicians. Association Rule Mining is employed to discover the prescription pattern and present the patterns as rules to support the prescription. To generate the interesting prescription rule set, Apriori algorithm proposed by Agrawal and Srikant (1994) is used. Before applying the algorithm, it is necessary to set the Minimum Support Count (minSC) as a parameter to qualify the validity of an item or itemset. There is no standard percentage for deciding the minSC whereas minSC is usually decided by users or experts (Zhang et al., 2004). To get a better minSC, it is essential to attempt more and give the result users for critique. After setting the value of minSC, the steps of Apriori algorithm are described as below.

Step 1. Find out the frequency of occurrence of different items in transactions, known as Support Counts (SC).

Step 2. Compare SC with the minSC. If the SC is smaller than the minSC, the corresponding items will be pruned.

Step 3. Combine the remaining items to form an itemset with 2 items.

Step 4. Scan the SC of the itemset in the transactional data.

Step 5. Compare the SC of itemset with minSC first. Then, prune off the itemset if the SC of itemset is smaller than the minSC.

Step 6. Generate itemset with 3 items. If itemsets contain pruned parent itemset(s), the itemsets are pruned too.

Step 7. Scan the transactional data for SC of each of the remaining itemset(s).

Step 8. Compare the SC of itemset with the minSC. Repeat the steps until the SC is equal to or less than the minSC. 
Step 9. Calculate the confidence and improvement (Bayardo et al., 2000). Normally, if confidence is greater than $90 \%$ and improvement greater than 1 . The result is good and suitable for prediction.

3.4 Data Tier: The Data Tier contains several data warehouses to store and manage the information of iEMRS. Raw data are converted to meaningful information in Logic Tier after the data collection and hence stored into corresponding databases in the Data Tier. All the required information is shown in the Presentation Tier via the secure data communication of the Communication Tier.

\section{System Evaluation and Discussion}

To evaluate the knowledge generated by association rule mining, hit rate is calculated. Hit rate is the number of drugs in prescription which match the drugs generated by association rules out of the number of drugs prescribed to patients. The formula of measuring hit rate is shown as below.

Hit Rate $=\frac{\text { No. of drugs suggested by the association rule mining }}{\text { No. of drugs actually selected by the physician }}$

In this study, 100 prescription records (i.e. collected from a Hong Kong medical organization) (Ting et al., 2011) were used as the training set in the association rule mining to discover the patterns in the drug utilization in prescription. Then, another 35 prescription records is used for testing and evaluating the validity of the system. According to Table 1, physicians prescribed four drugs in average, the hit rate will be valid if 3 or more out of 4 drugs of prescription exist in the result of association rule mining. This implies that the hit rate is regard as valid if it is equal to or more 0.75 .

As shown in Figure 3,60\% of the records achieve hit rate (with a value of 1 ) when the minSC set to 6. About $85 \%$ of the records achieve hit rate equal to or greater than 0.75 . These results represent that physicians can choose most of the drugs in the result of association rule mining when they determine the treatment. In other words, the proposed system can generate more relevant prescription decision(s) for physicians to encounter different patients' complaints whereas the suggested medicine in the decision support is nearly the same as the decision of physicians. Other than the high degree of similarity of medicine suggestion, it is also believed that the approach of knowledge capturing from other physicians' prescription behaviors is useful for physicians in the medicine selection process. In the case of medical prescription, physicians rely heavily on their knowledge and experiences to select appropriate medicine. It is almost impossible for a physician to utilize only their individual knowledge to consider all the important differences between current and former similar cases. Therefore, it is important to learn from others and consider other physicians' experience in making a prescription decision. 
Table 1. Average amount of drugs in prescription

\begin{tabular}{|c|c|c|c|c|}
\hline & Max & Min & Mean & Std Dev. \\
\hline Training Set (100 records) & 7 & 3 & 4.76 & 0.96 \\
\hline Testing Set (35 records) & 6 & 3 & 4.43 & 0.70 \\
\hline
\end{tabular}

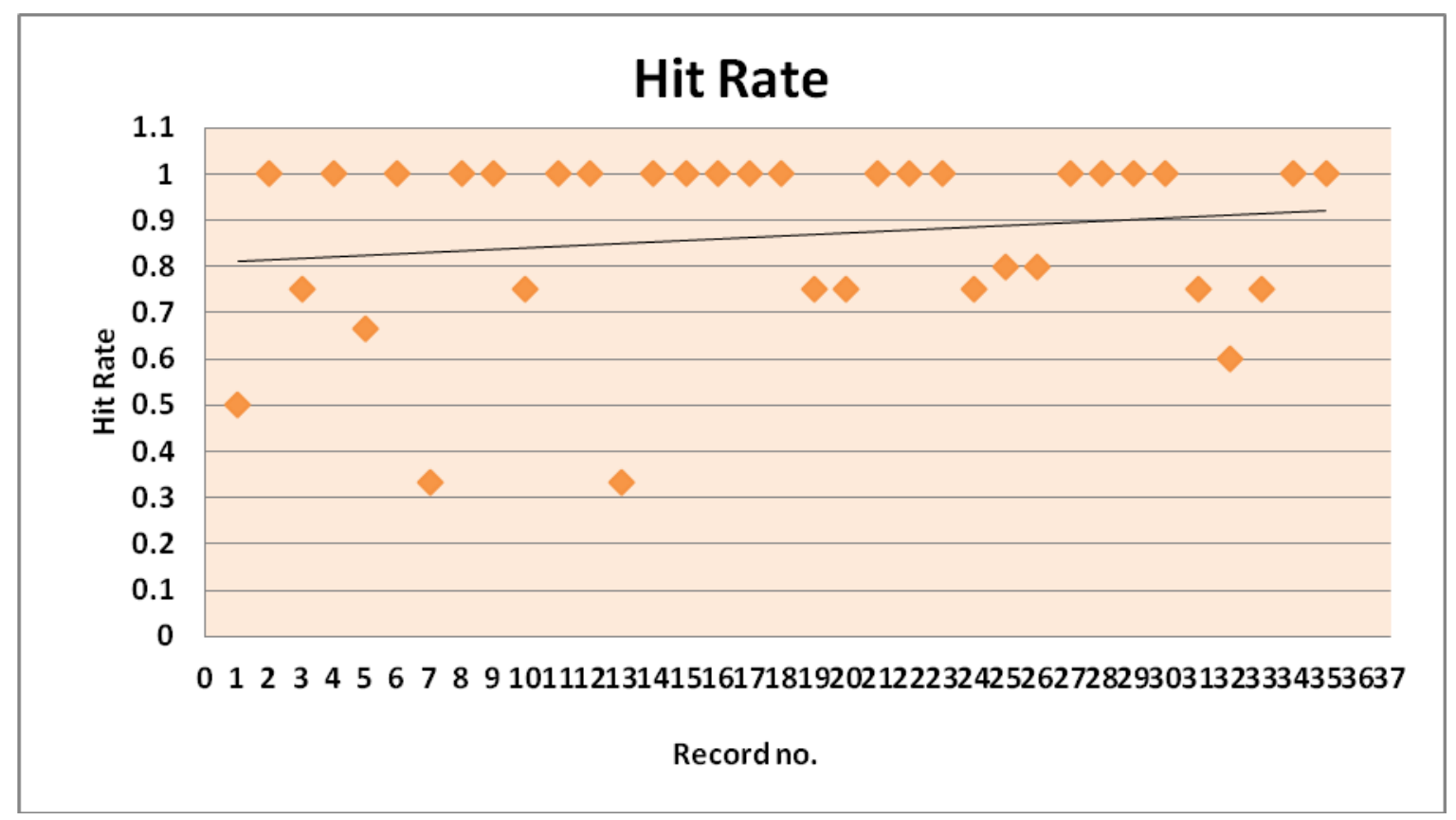

Figure 3. Hit rate of iEMRS

With the high satisfactory level in the medicine recommendation of iEMRS, this study also measures the efficiency of iEMRS in practice. A case study has been conducted in collaboration with HUMP (Anonymous name). Prior to the implementation of iEMRS, paper-based records system was applied in the HUMP. The time of the procedure (i.e. from the patient registration to the end of drug dispensing) is about 20 to 30 minutes; whereas after the implementation of iEMRS, the entire process reduces to 12 - 19 minutes. Table 2 depicts the responsibilities of physicians and nurses in HUMP before and after the implementation of iEMRS. According to the results, the workload of nurses and physician is reduced as well as the time required to perform the tasks is saved significantly.

In case of the nurses, iEMRS make their works more efficient. When a patient visits the clinic, nurse can assess to patient's information directly and instantly since the information (stored in the system) is completed and systematically managed. As a result, the process of registration and checking patient's information can be completed in 2 minutes. Furthermore, the nurse does not require to find the paper-based medical records from shelves, which in turn of saving for 5 minutes. Last but not least, the nurse can pack the drugs under clear guidelines. Therefore, the speed of drug packaging is faster and the mispacking errors are greatly reduced. 
In addition to nurses' operations, the medical knowledge in iEMRS assists physicians to make better prescription decision. With instant referencing to the comprehensive past medical histories of the patients and drug list recommendation (through discovering the pattern in the database), physicians agreed that the medicine selection process can greatly improved, especially in the area of drug-drug interaction checking.

Table 2. Responsibilities and times required (before and after the implementation of iEMRS)

\begin{tabular}{|c|c|c|c|c|c|c|}
\hline \multirow[b]{2}{*}{ Responsibility } & \multicolumn{3}{|c|}{ Before the system adoption } & \multicolumn{3}{|c|}{$\begin{array}{l}\text { After the implementation of } \\
\text { iEMRS }\end{array}$} \\
\hline & Nurse & Physicians & $\begin{array}{c}\text { Time } \\
\text { Required } \\
\text { (mins) }\end{array}$ & Nurse & Physicians & $\begin{array}{c}\text { Time } \\
\text { Required } \\
\text { (mins) }\end{array}$ \\
\hline $\begin{array}{l}\text { Registration for } \\
\text { consultation }\end{array}$ & $\checkmark$ & & 1 & $\checkmark$ & & 0.5 \\
\hline $\begin{array}{l}\text { Checking and validating } \\
\text { of patients' information }\end{array}$ & $\checkmark$ & & $1-3$ & $\checkmark$ & & $1-1.5$ \\
\hline $\begin{array}{l}\text { Search of paper-based } \\
\text { records from cabinets }\end{array}$ & $\checkmark$ & & 5 & & & 0 \\
\hline Consultation & & $\checkmark$ & $3-5$ & & $\checkmark$ & $3-5$ \\
\hline $\begin{array}{l}\text { Prescription (i.e. medicine } \\
\text { selection/determination) }\end{array}$ & & $\checkmark$ & $3-5$ & & $\checkmark$ & $2-3$ \\
\hline $\begin{array}{l}\text { Drug packing (i.e. packing } \\
\text { the drugs from big } \\
\text { container to small } \\
\text { container) }\end{array}$ & $\checkmark$ & & $5-7$ & $\checkmark$ & & $3-5$ \\
\hline $\begin{array}{l}\text { Billing and explanation of } \\
\text { prescription instruction to } \\
\text { the patient }\end{array}$ & $\checkmark$ & & $3-4$ & $\checkmark$ & & $3-4$ \\
\hline
\end{tabular}

\section{Conclusions}


Advert cases in prescription cause long term effect to the patients. Prescription errors exist over the world every day. The problems raise alarm to the medical industry and government. Although EMRS can reduce prescription errors, the problems still perplex medical organization. The traditional EMRS can merely assist data recording aspects; whereas it is not adequate to enhance the process of prescription. Therefore, EMRS with decision support capability in medical prescription is necessary for medical organizations.

In this study, iEMRS is proposed to enhance the decision support capability in the prescription process. Medical knowledge is consolidated by scientific techniques and shown under systematical methods. Physicians can get knowledge from other physicians' prescription behaviors during the medicine selection process. Therefore, the final decision by physicians will be enhanced after taking more physicians' consideration, so as to improve the patient safety by reducing the medication errors.

One limitation of this study is the small sample size of the records for system evaluation. To provide a complete assessment of the system, the results should be examined in combination with the medical records obtained at other organizations such as hospitals (as the current medical records are collected from one medical organization). Due to the limited medical records collected, it is expected that more experiments are conducted with different setting of minSC in further work so as to determine the optimal value of minSC. Furthermore, the present study is applied to text-based medical information. The integration of multimedia such as medical images and sound effects is needed. It will be an interesting area to investigate the integration of visual and aural media along with written medical records. For example, adoption of speech recognition technique is one of the possible directions to capture the conservation of patients and physicians for better informing the clinical decisions.

\section{Acknowledgement}

Acknowledgement is given to Dr. Peter Lo, Dr. Francis Liu, Dr. C.W. Lo and Miss Maggie Poon for their guidance on issues in clinical coding and medical knowledge in general. The authors would also like to express their sincere thanks to the Research Committee of the Hong Kong Polytechnic University for providing the financial support for this research work.

\section{References}

Agrawal R. and Srikant R. 1994. Fast algorithms for mining association rules in large databases, Proceedings of the 20th International Conference on Very Large Data Bases, VLDB, pp. 487-499. Bayardo R.J., Agrawal R. and Gunopulos D. 2000. Constraint-based rule mining in large, dense databases, Data Mining and Knowledge Discovery, Vol. 4, No. 2-3, pp. 217-240. 
Cheung N.T., Fung K.W., Wong K.C., Cheung A., Cheung J., Ho W., Cheung C., Shung E., Fung V. and Fung H. 2001. Medical informatics the state of the art in the Hospital Authority, International Journal of Medical Informatics, Vol. 62, No. 2-3, pp.113-119.

Condren M., Studebaker I.J. and John B.M. 2011. Prescribing errors in a pediatric clinic, Clinical Pediatrics, Vol. 49, No. 1, pp. 49-53.

Holliday I. and Tam W.K. 2004. E-health in the East Asian tigers, International Journal of Medical Informatics, Vol. 73, No. 11, pp. 759-769.

Hu P.J., Wei C.P. and Cheng T.H. 2002. Investigating telemedicine developments in Taiwan: implications for telemedicine program management, IEEE Proceedings of the 35th Hawaii International Conference on System Sciences, pp.8.

Khanapi M., Bali R.K., Naguib N.G. and Marshall I.M. 2008. Electronic health records approaches and challenges: a comparison between Malaysia and four East Asian countries, International Journal of Electronic Healthcare, Vol. 4, No. 1, pp. 78-104.

Randolph A.G., Haynes R.B., Wyatt J.C., Cook D.J. and Guyatt G.H. 1999. Users' guides to the medical literature: XVIII. how to use an article evaluating the clinical impact of a computer-based clinical decision support system, Journal of the American Medical Association, Vol. 282, No. 1, pp. 67-74.

Regnstrom K. and Burgess D.J. 2005. Pharmacogenomics and its potential impact on drug and formulation development, Critical Reviews in Therapeutic Drug Carrier Systems, Vol. 22, No. 5, pp. 465-492.

Roukema J., Los R.K., Bleeker S.E., Van Ginneken A.M., Van der Lei J. and Moll H.A. 2006. Paper versus computer: feasibility of an electronic medical record in general pediatrics, Pediatrics, Vol. 117, No. 1, pp. 15-21.

Sánchez J.L., Savin S. and Vasileva V. 2005. Key success factors in implementing electronic medical records in University Hospital of Rennes, L’Ecole Nationale de la Santé Publique (National School of Public Health), Rennes, Rennes, France.

Teich J.M., Osheroff J.A., Pifer E.A., Sittig D.F. and Jenders R.A. 2005. Clinical decision support in electronic prescribing: recommendations and an action plan: report of the joint clinical decision support workgroup, Journal of the American Medical Informatics Association, Vol. 12, No. 4, pp. 365-376.

Ting S.L., Kwok S.K., Tsang A.H.C. and Lee W.B. 2010. CASESIAN: A knowledge-based system using statistical and experiential perspectives for improving the knowledge sharing in the medical prescription process, Expert Systems With Applications, Vol. 37, No. 7, pp. 5336-5346.

Ting S.L., Kwok S.K., Tsang A.H.C. and Lee W.B. 2011. A hybrid knowledge-based approach to supporting the medical prescription for general practitioners: Real case in a Hong Kong medical center, Knowledge-Based Systems, Vol. 24, No. 3, pp. 444-456.

Toyoda K. 1998. Standardization and security for the EMR, International Journal of Medical Informatics, Vol. 48, No. 1-3, pp.57-60. 
Kozier B.J., Erb G., Berman A.J. and Snyder S. 2004. Fundamentals of nursing: concepts, process, and practice, Upper Saddle River, New Jersey, Prentice Hall Health.

Wyatt J.C. 2000. Decision support systems, Journal of the Royal Society of Medicine, Vol. 93, No. 11, pp.629-33.

Zhang S.C., Zhang C.Q. and Wu X.D. 2004. Knowledge Discovery in Multiple Databases, Springer. 